\title{
Community matters
}

\author{
We publish in this issue our first Matters Arising, a new way for Nature Research journals to host a sound and \\ peer-reviewed debate driven by the community on a stimulating (and maybe polarizing) topic presented in a \\ published paper.
}

T his is a celebratory month for space exploration. It marks, of course, the 50th anniversary of the Moon landing, a milestone in humankind's push beyond our Earth. This issue showcases several examples of the progress we have made during these 50 years, from groundbased (Letter by Sinclair et al. and Letter by Lellouch et al.) and space-based (Article by Griffith et al. and Article by Tosi et al.) astronomical facilities to innovative techniques for analysing current and past datasets, such as deep learning (Letter by Waldmann and Griffith). This issue also contains a Perspective that summarizes humankind's first close-up view of an interstellar body, 'Oumuamua, which tested the capabilities of the astronomy community to reach to a very faint and unusual object that was moving very quickly away from us.

And what better way to celebrate the Moon landing than by selecting new space exploration missions? Several announcements have been made recently. NASA's Artemis project to go back to the Moon by 2024 is getting more defined at a rapid pace. NASA also recently shortlisted three deep-space smallsats (binary asteroids, Mars atmospheric escape and lunar water mapping are the lucky ones). At the end of June, the European Space Agency selected Comet Interceptor, a new type of mission that will lurk at the L2 Lagrange point waiting for a suitable target in the form of a pristine comet or interstellar object. Just one week later, NASA selected Dragonfly, a rotorcraft lander for the in situ exploration of different regions on Titan, as the next spacecraft in the New Frontiers programme, to which belong some of the most successful recent NASA missions (New Horizons, Juno, OSIRIS-REx).

A complementary way to explore our surroundings is to study extraterrestrial bodies that fell on Earth: meteorites. This issue presents two papers using meteorites for two completely different scientific objectives, highlighting the versatility of this 'exploration' technique. In a Letter, Pierre Haenecour and colleagues study the composition of a grain embedded into a meteorite to advance our knowledge of novae. In an Article, Larry Nittler and colleagues report an inclusion within a primitive meteorite, which they associate with a cometary planetesimal, revealing information on the transport of material between the outer and the inner Solar System during its formation.

The Nittler et al. Article is also connected to our first Matters Arising, a new content type that aims to provide an avenue for the community to comment on issues of a paper we have recently published, using the same editorial engagement we employ for standard research papers. Matters Arising was introduced at Nature and the other Nature Research journals a few months ago (Nature 562, 460; 2018).

A paper's story does not stop at publication, as it can generate debate and even controversy well beyond its publication date. Yet, the current editorial process, largely based on a succession of collected papers, struggles to include such debate. Usually, the natural way is to publish another paper on the issue. However, as a standard paper needs to contain original research and have a more global approach, it cannot focus on debating a specific point (or set of points) of another paper.

As this Matters Arising represents a first for our journal, it is instructive to look at it in some detail to illustrate the process and highlight with a practical example the role that such content fulfils. Let's start with the paper from which the Matters Arising stems. Nittler et al. present the discovery of a grain of cometary origins within a stony meteorite. One of the pieces of evidence brought forward by Nittler et al. for the identification of the grain as being akin to pristine cometary material is the identification of some specific inclusions called GEMS (glass with embedded metal and sulfides). The author of the Matters Arising, John Bradley, while not negating the main discovery of the Nittler et al. paper, found the identification of GEMS unconvincing and proposed to write a Matters Arising on his point of view.

This part already highlights the purpose of Matters Arising: it is not a request for correction of a mistake, but a scientific comment, point of view or clarification on a specific point included in a published paper. Another important point is its timely nature. The matter under debate must be a current issue that the community recognizes as interesting and widely discussed. This assessment is firstly an editorial call, inferred by recent publications and conference presentations. In this specific case, the nature of GEMS has been debated among the reviewers of the paper, so it was already evident that it was an unsolved issue. To facilitate the immediacy of the debate, we also encourage authors to make Matters Arising available on a preprint repository such as arXiv. As a Matters Arising is peer-reviewed, one or more experts are consulted, providing additional feedback on the pertinence of the manuscript and of the interest of the matter at hand. This process ensures that the community is the main target, subject and beneficiary of the Matters Arising formula.

Another important part is communication. The purpose of Matters Arising is not the rebuttal per se of a point of a paper, but the highlighting of an issue that the author thinks should be brought forward to the community. To encourage an open and respectful debate, the author of the Matters Arising is invited to contact the corresponding author of the paper before submitting the manuscript to us, to discuss the situation in private. Only when the differences cannot be resolved do we recommend the Matters Arising route, and the published paper's authors are also invited to draft a reply. The reply is also assessed by a reviewer or reviewers, who advise on the suitability of the publication of the Matters Arising, of the reply, or both. Every exchange (except those between the author of the Matters Arising and the author of the paper) is overseen by Nature Astronomy editors.

In the end, we hope Matters Arising will open up constructive dialogue for advancing knowledge and will be warmly adopted by our community.

Published online: 5 July 2019

https://doi.org/10.1038/s41550-019-0851-7 